\title{
Significance of CD47 expression in gastric cancer
}

\author{
TOMOYA SUDO ${ }^{1,2}$, YUSUKE TAKAHASHI ${ }^{3}$, GENTA SAWADA ${ }^{3}$, \\ RYUTARO UCHI $^{2}$, KOSHI MIMORI ${ }^{2}$ and YOSHITO AKAGI ${ }^{1}$
}

\author{
${ }^{1}$ Department of Surgery, Kurume University School of Medicine, Kurume, Fukuoka 830-0011; ${ }^{2}$ Department of \\ Surgery and Molecular Oncology, Medical Institute of Bioregulation, Kyushu University, Beppu, Oita 847-0838; \\ ${ }^{3}$ Department of Surgical Oncology, Osaka University, Graduate School of Medicine, Suita, Osaka 565-0871, Japan
}

Received October 5, 2015; Accepted March 7, 2017

DOI: $10.3892 / 01.2017 .6257$

\begin{abstract}
Integrin-associated protein (CD47) is ubiquitously expressed on the surface of cells and functions as an identifier of self. In blood cancer, tumor cells expressing CD47 evade phagocytosis by macrophages, leading to a poor patient prognosis. However, the status of CD47 expression in solid tumors, particularly in gastric cancer, is not well understood. The purpose of the present study was to examine the level of CD47 in the primary tumor, peripheral blood (PB) and bone marrow $(\mathrm{BM})$ of patients with gastric cancer, and to determine its effect. Reverse transcription-quantitative polymerase chain reaction analysis was performed to determine the level of CD47 mRNA expression in primary tumor, PB and BM samples collected from 168 patients with gastric cancer. Cell sorting was performed to investigate CD47 protein expression in $\mathrm{PB}$ and BM fractions, and to identify the source of CD47 expression. In primary tumors, the expression of CD47 was not associated with any clinicopathological factors or prognosis. By contrast, in PB, the low CD47 expression group demonstrated a significantly increased tumor size, and frequency of lymphatic invasion and lymph node metastasis, compared with the high CD47 expression group. In addition, the clinical tumor stage of the low CD47 expression group was significantly increased compared with that of the high CD47 expression group. Conversely, in PB, the high CD47 expression group had a significantly higher frequency of lymphatic invasion and lymph node metastasis compared with the low CD47 expression group.
\end{abstract}

Correspondence to: Dr Tomoya Sudo, Department of Surgery, Kurume University School of Medicine, 67 Asahi, Kurume, Fukuoka 830-0011, Japan

E-mail: sudou_tomoya@med.kurume-u.ac.jp

Abbreviations: CD47, integrin-associated protein; RT-PCR, reverse transcription-polymerase chain reaction; CD14, myeloid cell-specific leucine-rich glycoprotein; CD45, protein tyrosine phosphatase receptor type C; CD325, neural cadherin

Key words: CD47, gastric cancer, peripheral blood, bone marrow, cell fraction
The lymphocyte fraction exhibited the highest CD47 expression compared with the other fractions in PB and BM samples. Low expression of CD47 was associated with the advancement of gastric cancer, in contrast to other cancers, and it may be associated with a decrease in lymphocytes during later stages. These results indicate that CD47 expression in the PB and BM may serve as a marker to analyze the immunological function of patients with gastric cancer; however, the significance of CD47 in gastric cancer requires further study.

\section{Introduction}

In the United States, the incidence of gastric cancer is not particularly high compared with that observed in other countries; however, 21,600 patients were diagnosed with gastric cancer in 2013, and the 5-year survival rate for all stages of the disease was only $27 \%$ (1). The disease is also a leading cause of cancer-associated mortality in males and females in Japan (2). The prognosis for patients with gastric cancer has gradually improved over the last 10 years; however, the 5-year survival rate of metastasized gastric cancer remains at $\sim 40 \%$ (3). Therefore, the identification and development of therapeutics is required to improve the prognosis of patients with advanced gastric cancer.

Integrin associated protein (CD47) is a glycoprotein that is ubiquitously expressed on the plasma membrane of all hematopoietic cells and the majority of other cell types $(4,5)$. Oldenborg et al (6) revealed that CD47 is a marker of self on murine red blood cells (RBCs), and that CD47-negative RBCs are rapidly eliminated from the circulation by macrophage phagocytosis. Furthermore, tumor cells expressing CD47 evade elimination by macrophages (7). The clinical significance of CD47 expression in blood cancer has been extensively studied (6-8). In solid tumors, including bladder cancer, cells expressing CD47 were identified to be of a tumor-initiating cell population (9). In addition, the prognosis of patients with breast cancer with high levels of CD47 expression was significantly poorer compared with patients with low expression of CD47 (10). In 2015, it was revealed that in addition to being associated with macrophage phagocytosis, a CD47 blockade drives the T-cell mediated elimination of immunogenic tumors (11). Therefore, CD47 is emerging as a focus of the field of immunotherapy.

Targeting CD47 via anti-CD47 antibody therapy was recently proposed (12). A variety of cancer types may be 
targeted with this therapy, and therefore, the status of CD47 expression requires investigation in various malignant tumors. However, little is known about the status of CD47 expression in patients with gastric cancer. Thus, the present study aimed to determine the level of CD47 expression in the primary tumor, peripheral blood $(\mathrm{PB})$ and bone marrow $(\mathrm{BM})$ of patients with gastric cancer, and to explore the potential of anti-CD47 antibody therapy in gastric cancer.

\section{Materials and methods}

Patients. A total of 168 patients (108 male, 60 female; mean age, $69 \pm 11.8$ years) who were diagnosed with gastric cancer and treated with gastrectomy, including lymphadenectomy, at Kyushu University Beppu Hospital (Oita, Japan) between April 2000 and April 2005 were enrolled in the present study. Written informed consent was obtained from all patients according to the guidelines provided by the Institutional Research Board of Kyushu University Beppu Hospital, which approved the present study. None of the patients received pre-surgical chemotherapy or radiotherapy. Pathological diagnosis and disease staging were performed according to the criteria of the Japanese Classification of Gastric Carcinoma (13). All patients were monitored closely for the recurrence of cancer following surgery every 3 months for 3-5 years.

Sample collection. PB samples were obtained from 6 healthy volunteers and the 168 patients with gastric cancer prior to surgery. PB samples $(10 \mathrm{ml})$ were taken and mixed with $4 \mathrm{ml}$ ISOGEN-LS reagent (Nippon Gene Co., Ltd., Toyama, Japan) per $1 \mathrm{ml}$ whole blood. The sample was stored for $5 \mathrm{~min}$ at room temperature, then snap-frozen immediately in liquid nitrogen and stored at $-80^{\circ} \mathrm{C}$ until required for RNA extraction.

BM samples were obtained from 160 patients with gastric cancer under general anesthesia prior to surgery. BM samples were taken from the sternum using a 15-gauge needle. Since there was a possibility of aspirating skin with the BM samples, the first $1 \mathrm{ml}$ of the sample was discarded, and another syringe was used to aspirate 20-30 $\mathrm{ml}$ of BM. ISOGEN-LS reagent was then added as previous, and the BM samples were subsequently processed in the same manner as the PB samples.

A total of 10 tumor and corresponding normal $4-\mu \mathrm{m}$ thick formalin-fixed paraffin-embedded tissue section samples from the patients who underwent surgery were also obtained and used for immunohistochemical analysis. A total of 6 autopsy bone marrow samples were collected, 3 from patients who succumbed to advanced gastric cancer, and 3 from pneumonia. Formalin-fixed paraffin-embedded $4-\mu \mathrm{m}$ tissue sections were made from these bone marrow samples.

$R N A$ preparation and reverse transcription $(R T)$. Total RNA was extracted from PB and BM samples using the ISOGEN-directed chloroform extraction and isopropanol precipitation protocol, as described previously (14). cDNA was synthesized using RT as described previously (15).

Cell lines. The human gastric cancer cell lines KATO-III, MKN-1, MKN-45 and MKN-74 were provided by the Cell Resource Center for Biomedical Research (Institute of Development, Aging and Cancer, Tohoku University, Sendai,
Japan). The NUGC-4 (JCRBO834) cell line was provided by the Japanese Collection of Research Bioresources Cell Bank (National Institutes of Biomedical Innovation, Health and Nutrition, Osaka, Japan). KATO-III cells were maintained in McCoy5A medium supplemented with $10 \%$ fetal bovine serum and $1 \%$ penicillin and streptomycin antibiotics (all from Thermo Fisher Scientific, Inc., Waltham, MA, USA). The other cell lines were maintained in an RPMI-1640 medium supplemented with $10 \%$ fetal calf serum and $1 \%$ penicillin and streptomycin (all from Thermo Fisher Scientific, Inc.). All cell lines were cultured in $10 \mathrm{~cm}$ culture dishes with $5 \% \mathrm{CO}_{2}$ at $37^{\circ} \mathrm{C}$. Cultured cells from each cell line were dissolved in $350 \mu 1$ of Buffer RLT containing $1 \% \beta$-mercaptoethanol, and total RNA was extracted and purified using the RNeasy Mini kit (Qiagen GmbH, Hilden, Germany) in accordance with the manufacturer's protocol.

Quantitative polymerase chain reaction ( $q P C R)$ evaluation of CD47 expression in clinical samples. The following primers were used to amplify CD47 from the RNA samples from the cells, PB and BM: Sense, 5'-GGCAATGACGAA GGAGGTTA-3' and antisense, 5'-ATCCGGTGGTATGGATG AGA-3'. GAPDH, used as an internal control, was amplified using the following primers: Sense, 5'-TGAACGGGAAGC TCACTGG-3' and antisense, 5'-TCCACCACCCTGTTGCT GTA-3'. qPCR was performed using the LightCycler ${ }^{\mathrm{TM}}$ system and SYBR-Green I dye (both from Roche Diagnostics, Indianapolis, IN, USA) according to the manufacturer's protocol. Briefly, each reaction contained $80 \mathrm{ng}$ cDNA, $2 \mu \mathrm{l}$ DNA Master SYBR-Green I mix, 50 ng primers and $2.4 \mu \mathrm{l}$ $25 \mathrm{mM} \mathrm{MgCl}_{2}$. The final volume was adjusted to $20 \mu \mathrm{l}$ with water. qPCR was performed with the following thermocycling conditions: Initial denaturation at $95^{\circ} \mathrm{C}$ for $10 \mathrm{~min}$; followed by 40 cycles of $95^{\circ} \mathrm{C}$ for $10 \mathrm{sec}$; annealing at $60^{\circ} \mathrm{C}$ for $10 \mathrm{sec}$; and extension at $72^{\circ} \mathrm{C}$ for $10 \mathrm{sec}$. The product was confirmed by gel electrophoresis. Following amplification, the products were subjected to a temperature gradient from $68-95^{\circ} \mathrm{C}$ at a rate of $0.2^{\circ} \mathrm{C} / \mathrm{sec}$, under continuous fluorescence monitoring, in order to produce a melt curve for each product. All concentrations were calculated relative to the concentration of the positive control, cDNA produced from Human Universal Reference Total RNA (Clontech Laboratories, Inc., Mountain View, CA, USA), using a previously described method (16). The negative control was water. Normalized expression values were obtained by dividing the mRNA concentration of CD47 by that of the internal control.

Immunohistochemistry. Formalin-fixed and paraffin-embedded tissue sections corresponding to the samples used for mRNA expression analysis were analyzed. Tissue sections were de-paraffinized, soaked in 0.01 M sodium citrate buffer and boiled in a microwave oven for $15 \mathrm{~min}$ at $500 \mathrm{~W}$ to retrieve cell antigens. In the case of bone marrow samples, decalcification was performed according to the manufacturer's protocol (Super Decalcifier I; Polyscience Inc., Warminster, PA, USA). The tissue sections were stained immunohistochemically using the streptavidin-biotin peroxidase method (Universal Dako Cytomation LSAB ${ }^{\circledR}$ kit; Dako; Agilent Technologies, Inc., Santa Clara, CA, USA) according to the manufacturer's protocol, with primary antibodies against CD47 (mouse 


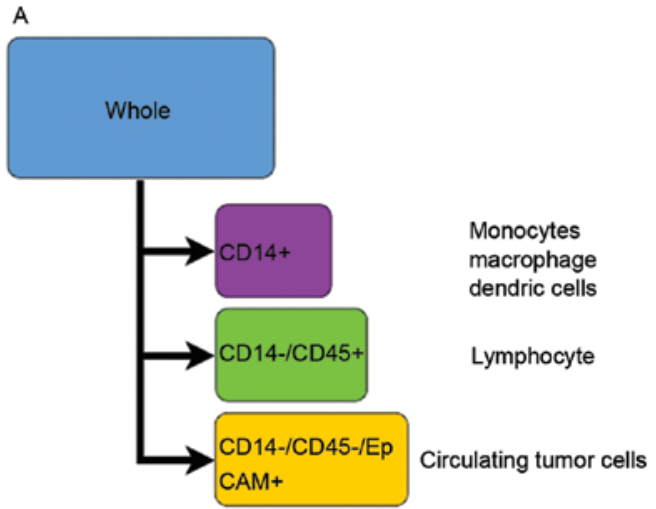

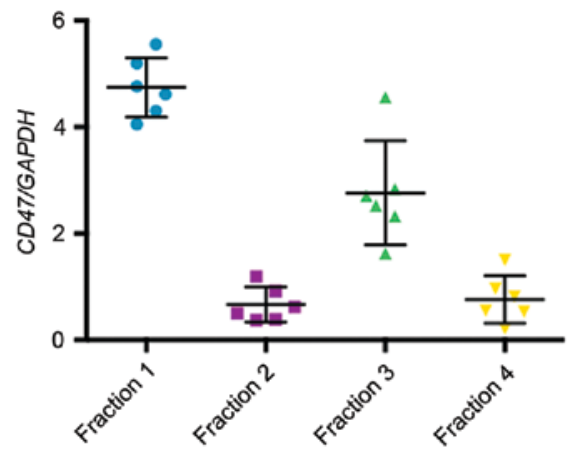

C

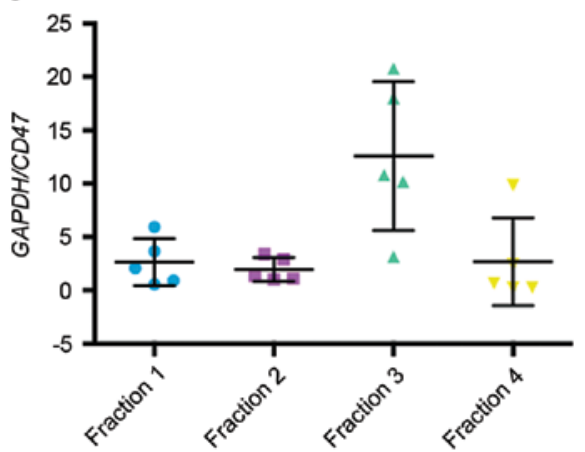

Figure 1. Schema of cell sorting and CD47 expression in peripheral blood and bone marrow. (A) Schema of the cell sorting procedure. (B) Comparison of CD47 mRNA expression in each fraction of peripheral blood. (C) Comparison of CD47 mRNA expression in each fraction of bone marrow. CD47, integrin-associated protein; CD14, myeloid cell-specific leucine-rich glycoprotein; CD45, protein tyrosine phosphatase receptor type C; EpCAM, epithelial cell adhesion molecule.

monoclonal antibody; dilution, 1:200; cat. no. sc-12730; Santa Cruz Biotechnology, Inc., Dallas, TX, USA). Briefly, the tissue sections were blocked with $3 \% \mathrm{H}_{2} \mathrm{O}_{2}$ for 5 min and incubated overnight with the primary antibody at $4^{\circ} \mathrm{C}$. The samples were then washed with TBS buffer and subsequently incubated with the secondary antibody from the LSAB ${ }^{\circledR}$ kit for $30 \mathrm{~min}$ at room temperature.

Cell sorting. Immunomagnetic cell sorting was performed on $30 \mathrm{ml}$ of $\mathrm{PB}$ collected from 6 healthy donors and $30 \mathrm{ml}$ BM collected from 6 patients with gastric cancer. Fractionation was performed using the autoMACS ${ }^{\circledR}$ Pro Separator (Miltenyi Biotec GmbH, Bergisch Gladbach, Germany) according to the manufacturer's protocol. Cells were prepared as follows: The PB or BM sample was loaded into $10 \mathrm{ml}$ of Ficoll density medium (GE Healthcare Life Sciences, Chalfont, UK) prepared in a $50 \mathrm{ml}$ polypropylene tube. Gradient centrifugation was subsequently performed at room temperature for $30 \mathrm{~min}$ at $450 \mathrm{x} \mathrm{g}$. Sample interfaces were collected subsequent to discarding the supernatants. The interface containing the mononuclear fractionated cells was washed twice with $20 \mathrm{ml}$ PBS and centrifuged at $150 \mathrm{xg}$ for $5 \mathrm{~min}$ at $4^{\circ} \mathrm{C}$.

Human CD326 [epithelial cell adhesion molecule (EpCAM)]-phycoerythrin MicroBeads (Miltenyi Biotec $\mathrm{GmbH}$ ) were used for positive selection of viable epithelial tumor cells from the samples, CD45-MicroBeads (Miltenyi Biotec $\mathrm{GmbH}$ ) were used to detect leukocytes and CD14-MicroBeads (Miltenyi Biotec $\mathrm{GmbH}$ ) were applied for the detection of monocytes and macrophages. The MicroBeads were used according to the manufacturer's protocol. In brief, the $\mathrm{CD} 14^{+}$fraction was sorted, then the $\mathrm{CD} 14^{-} / \mathrm{CD} 45^{+}$fraction and finally the EpCAM-positive fraction (Fig. 1A). The expression of CD47 in each fraction was measured using RT-qPCR as described above.

Statistical analysis. The association between CD47 mRNA expression and clinicopathological factors was evaluated using the $\chi^{2}$ test. For the evaluation of primary tumor tissue samples, the median CD47 mRNA expression value was used as the cut-off to divided patients into high CD47 expression and low CD47 expression groups. For PB and BM samples, the median value was calculated using all samples. Overall survival was calculated by the Kaplan-Meier method and the differences in survival between the groups were compared using the log-rank test. All tests were performed using JMP software version 12.2.0 (SAS Institute, Inc., Cary, NC, USA) and GraphPad Prism software version 6.0 h (GraphPad Software, Inc., La Jolla, CA, USA). $\mathrm{P}<0.05$ was considered to indicate a statistically significant difference.

\section{Results}

Expression of CD47 in primary gastric tumors. Immunohistochemical analysis was performed to investigate the protein expression level of CD47 in the tissues of gastric cancer and adjacent normal mucosa. In normal gastric mucosa, intracellular expression of CD47 protein was observed in the bottom layer of the gastric gland, which almost disappeared in the upper layer (Fig. 2A). In gastric tumor tissues, a varying pattern of 
A

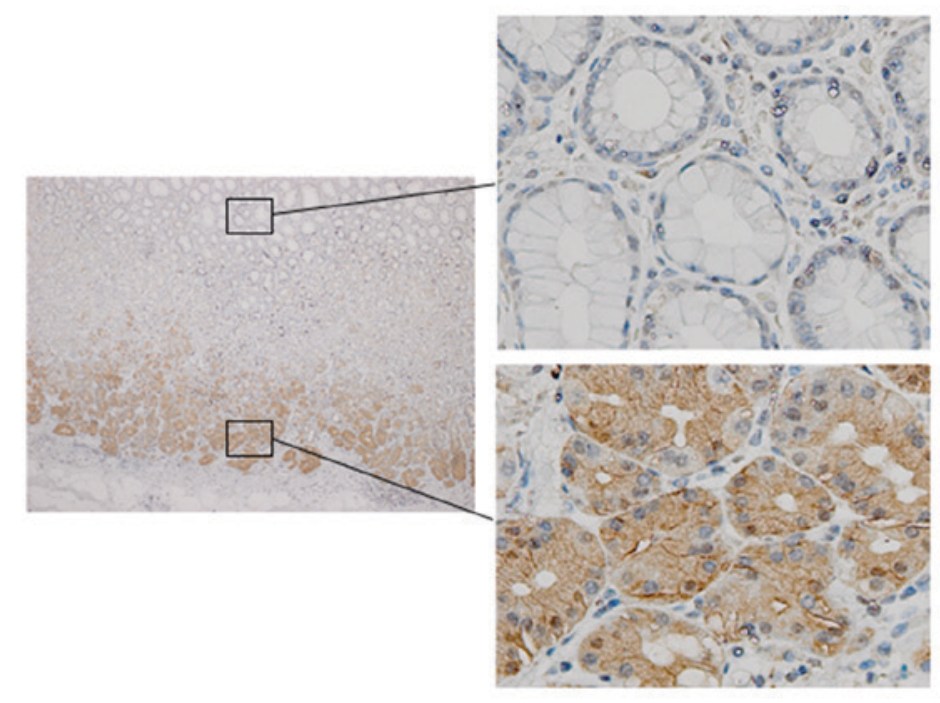

B

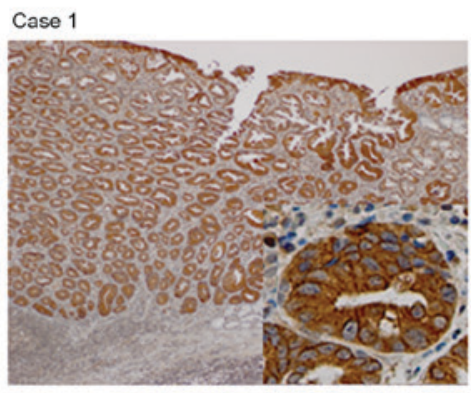

Case 2

Case 3

C

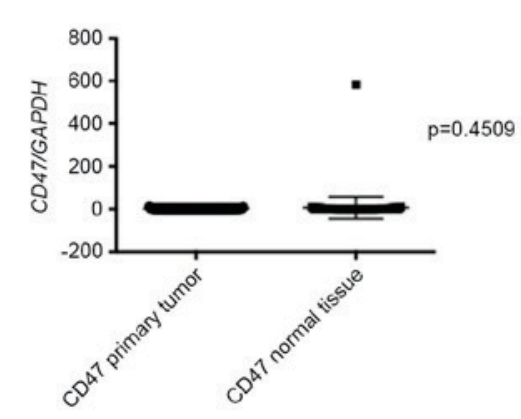

D

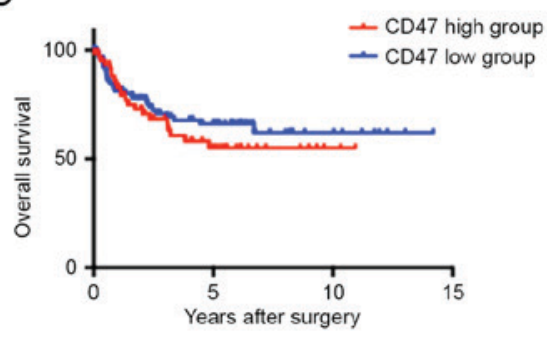

Figure 2. CD47 expression in primary tumor and normal mucosa tissues. (A) Immunohistochemical CD47 staining of normal mucosa of the stomach. Left panels, x10 magnification; right panels, x40 magnification. The right upper figure is the upper layer, and the right lower figure, the lower layer of the mucosa. (B) Immunohistochemical CD47 staining of gastric cancer tissues. Case 1 is well-differentiated adenocarcinoma, case 2 is moderately-differentiated adenocarcinoma and case 3 is poorly-differentiated adenocarcinoma. (C) Comparison of CD47 mRNA expression between primary gastric tumor tissue and normal gastric tissue. (D) Overall survival curves of the high and low CD47 expression groups. CD47, integrin-associated protein.

CD47 protein expression was observed. In one case, CD47 was highly expressed throughout the tissue, and this case was diagnosed as well-differentiated adenocarcinoma (Fig. 2B, case 1). In another case, the expression of CD47 was relatively high, and the expression pattern was not consistent throughout the tissue; this case was diagnosed as moderately-differentiated adenocarcinoma (Fig. 2B, case 2). The expression of CD47 nearly disappeared in another case, which was diagnosed as poorly-differentiated adenocarcinoma (Fig. 2B, case 3).

As demonstrated by immunohistochemical staining, CD47 protein levels appeared to be associated with the histological grade, and therefore, the CD47 mRNA expression level was compared with the histological grade; however, no significant difference was observed between CD47 expression and different histological grades (data not shown). The expression of CD47 was compared between gastric tumor tissue and corresponding normal tissue using RT-qPCR. However, as demonstrated in Fig. 2C, no significant difference was observed in CD47 mRNA expression between primary tumor tissue and normal tissue.

To determine the association between CD47 mRNA expression and the clinicopathological characteristics of patients with gastric cancer, patients were divided into two groups: High CD47 expression and low CD47 expression, however, no significant association was observed between CD47 expression and any of the clinicopathological factors tested (Table I). In addition, the overall survival rate between the high and low CD47 expression groups was compared, and no significant difference was observed between the two groups (Fig. 2D). 
Table I. Association between CD47 mRNA expression in tumor tissue and the clinicopathological characteristics of patients with gastric cancer.

CD47 expression group

\begin{tabular}{|c|c|c|c|}
\hline \multirow{2}{*}{ Clinicopathological characteristic } & & \multirow[b]{2}{*}{ P-value } \\
\hline & High & Low & \\
\hline Total, n & 65 & 68 & - \\
\hline Age, years, mean $\pm \mathrm{SD}$ & $64.74 \pm 1.37$ & $68.47 \pm 1.34$ & 0.0528 \\
\hline Age distribution, $\mathrm{n} \geq 70 /<70$ years $(\% \geq 70)$ & $27 / 38(41.6)$ & $29 / 39(42.6)$ & 0.897 \\
\hline Gender, male/female (\% male) & $42 / 23(64.6)$ & $42 / 26(61.7)$ & 0.733 \\
\hline \multicolumn{4}{|l|}{ Tumor factors } \\
\hline Tumor size, mm, mean \pm SD & $49.85 \pm 26.03$ & $53.79 \pm 34.19$ & 0.458 \\
\hline Tumor size distribution, $\mathrm{n} \geq 50 /<50 \mathrm{~mm}(\% \geq 50)$ & $31 / 34(47.6)$ & $39 / 29(57.3)$ & $0.0497^{\mathrm{a}}$ \\
\hline Depth of tumor, n T1-2/T3-4 (\% T1-2) & $43 / 22(66.2)$ & $46 / 22(67.7)$ & 0.855 \\
\hline Tumor type, $\mathrm{n}$ type $0-1 / 2-5(\%$ 0-1) & $13 / 52(20.0)$ & $21 / 47(30.9)$ & 0.149 \\
\hline Lymphatic invasion, $\mathrm{n}+/-(\%+)$ & $43 / 22(61.2)$ & $42 / 26(61.8)$ & 0.598 \\
\hline Vascular invasion, $\mathrm{n}+/-(\%+)$ & $19 / 46(29.2)$ & $14 / 54(20.6)$ & 0.248 \\
\hline \multicolumn{4}{|l|}{ Metastatic factors } \\
\hline Lymph node metastasis, $\mathrm{n}+/-(\%+)$ & $41 / 24(63.1)$ & $44 / 24(64.7)$ & 0.845 \\
\hline Liver metastasis, $\mathrm{n}+/-(\%+)$ & $2 / 63(3.1)$ & $6 / 62(8.8)$ & 0.154 \\
\hline Peritoneal (micro) metastasis, $\mathrm{n}+/-(\%+)$ & $9 / 56(13.9)$ & $9 / 59(13.2)$ & 0.918 \\
\hline Peritoneal (macro) metastasis, $\mathrm{n}+/-(\%+)$ & 9/56 (13.9) & 9/59 (13.2) & 0.918 \\
\hline Distant metastasis, $\mathrm{n}+/-(\%+)$ & $1 / 64(1.5)$ & $4 / 64(5.9)$ & 0.173 \\
\hline Clinical stage, n I/II-IV (\% I) & $21 / 44(32.3)$ & 27/41 (39.7) & 0.374 \\
\hline
\end{tabular}

${ }^{\mathrm{a}} \mathrm{P}<0.05$. CD47, integrin-associated protein; $\mathrm{SD}$, standard deviation.

Expression of $C D 47$ in the $P B . \mathrm{CD} 47$ expression in $\mathrm{PB}$ was analyzed, and its association with the clinicopathological characteristics of patients with gastric cancer was investigated (Table II). Tumor size and depth were significantly increased in patients with low compared with high CD47 expression. The frequency of lymphatic invasion was also significantly higher in the low compared with high CD47 expression group. The percentage of tumor type 2-5 was significantly higher at $73 \%$ in the CD47 low expression group compared with $44 \%$ in the CD47 high expression group. The frequency of lymph node metastasis and lymphatic invasion in the low CD47 expression group was significantly increased compared with that in the high CD47 expression group. As a result, the clinical tumor stage of the low CD47 expression group was significantly increased compared with that of the high CD47 expression group. The expression level of CD47 mRNA was compared with the clinical tumor stage in the PB sample (Fig. 3A). CD47 expression was demonstrated to be highest in tumor stage I in PB samples (Fig. 3A).

Expression of CD47 in the BM. CD47 protein expression in BM samples was analyzed using immunohistochemical staining. As demonstrated in Fig. 4, CD47 expression was markedly increased in the BM of patients with stage IV gastric cancer. To assess the importance of CD47 expression in the BM, the clinicopathological characteristics of patients with gastric cancer who exhibited high or low CD47 mRNA expression were compared. As indicated in Table III, the percentages of lymphatic invasion and lymph node metastasis were significantly higher in the high CD47 expression group compared with the low CD47 expression group. By contrast, the frequency of distant metastasis was significantly higher in the low CD47 expression group compared with the high CD47 expression group. In addition, the clinical tumor stage was significantly increased in the high compared low CD47 expression group. The BM expression of CD47 mRNA was significantly higher in stage III/IV patients than in stage I/II patients (Fig. 3B).

Origin of $C D 47$ in the $P B$ and $B M$. The results of the analysis of the association between CD47 mRNA expression and the clinicopathological characteristics of patients with gastric cancer were different in primary tumors, $\mathrm{PB}$ and BM. To detect the original source of CD47 expression in PB and BM, cell sorting was performed. $\mathrm{PB}$ and $\mathrm{BM}$ samples were sorted into the following four fractions (Fig. 1A): Fraction 1, whole blood cells; fraction 2 [myeloid cell-specific leucine-rich glycoprotein $(\mathrm{CD} 14)^{+}$cells], macrophages or monocytes; fraction 3 (CD14/CD $45^{+}$cells), lymphocytes; and fraction 4 (CD14/CD45 $/ \mathrm{EpCAM}^{+}$cells), circulating tumor cells. Prior to the analysis, CD47 expression was confirmed in 4 gastric cancer cell lines by RT-qPCR (data not shown). The expression of CD47 mRNA in each fraction is demonstrated in Fig. 1B and C. CD47 mRNA expression was expected to be highest in the circulating tumor cell fraction; however, the fraction expressing CD47 mRNA was larger in the PB (Fig. 1B) and BM (Fig. 1C) samples than the lymphocyte fraction. 
Table II. Association between CD47 mRNA expression in peripheral blood and the clinicopathological characteristics of patients with gastric cancer.

\begin{tabular}{lccc}
\hline & \multicolumn{2}{c}{ CD47 expression group } & \\
\cline { 2 - 3 } Clinicopathological characteristic & High & Low & P-value \\
\hline Total, $\mathrm{n}$ & 86 & 88 & - \\
Age, years, mean \pm SD & $59.45 \pm 1.32$ & $61.76 \pm 1.26$ & 0.207 \\
Age distribution, $\mathrm{n} \geq 70 /<70$ years $(\% \geq 70)$ & $18 / 68(20.9)$ & $26 / 62(29.5)$ & 0.460 \\
Gender, male/female $(\%$ male) & $59 / 27(68.6)$ & $55 / 33(62.5)$ & 0.397 \\
Tumor factors & & & \\
Tumor size, mm, mean \pm SD & $63.29 \pm 44.71$ & $82.58 \pm 44.45$ & $0.0025^{\mathrm{a}}$ \\
Tumor size distribution, $\mathrm{n} \geq 50 /<50$ mm $(\% \geq 50)$ & $38 / 48(44.1)$ & $61 / 27(69.3)$ & $0.0052^{\mathrm{a}}$ \\
Depth of tumor, $\mathrm{n}$ T1-2/T3-4 $(\%$ T1-2) & $37 / 49(43.0)$ & $7 / 81(7.9)$ & $<0.0001^{\mathrm{a}}$ \\
Tumor type, $\mathrm{n}$ type $0-1 / 2-5(\%$ - 1$)$ & $48 / 38(55.8)$ & $23 / 65(26.1)$ & $0.0001^{\mathrm{a}}$ \\
Lymphatic invasion, $\mathrm{n}+/-(\%+)$ & $47 / 39(54.6)$ & $63 / 25(71.6)$ & $0.00316^{\mathrm{a}}$ \\
Vascular invasion, $\mathrm{n}+/-(\%+)$ & $20 / 66(23.2)$ & $63 / 25(71.6)$ & 0.278 \\
Metastatic factors & & & \\
Lymph node metastasis, $\mathrm{n}+/-(\%+)$ & $45 / 41(52.3)$ & $71 / 17(80.6)$ & $0.0002^{\mathrm{a}}$ \\
Liver metastasis, $\mathrm{n}+/-(\%+)$ & $1 / 85(1.1)$ & $3 / 85(3.4)$ & 0.324 \\
Peritoneal $($ micro $)$ metastasis, $\mathrm{n}+/-(\%+)$ & $11 / 75(14.7)$ & $20 / 68(22.7)$ & 0.0868 \\
Peritoneal (macro) metastasis, $\mathrm{n}+/-(\%+)$ & $7 / 79(8.1)$ & $11 / 77(12.5)$ & 0.340 \\
Distant metastasis, $\mathrm{n}+/-(\%+)$ & $2 / 84(2.3)$ & $1 / 87(1.1)$ & 0.527 \\
Clinical stage, $\mathrm{n} \mathrm{I} / \mathrm{II}-\mathrm{IV}(\% \mathrm{I})$ & $45 / 41(52.1)$ & $9 / 79(10.2)$ & $<0.0001^{\mathrm{a}}$
\end{tabular}

${ }^{\mathrm{a}} \mathrm{P}<0.05$. CD47, integrin-associated protein; $\mathrm{SD}$, standard deviation.
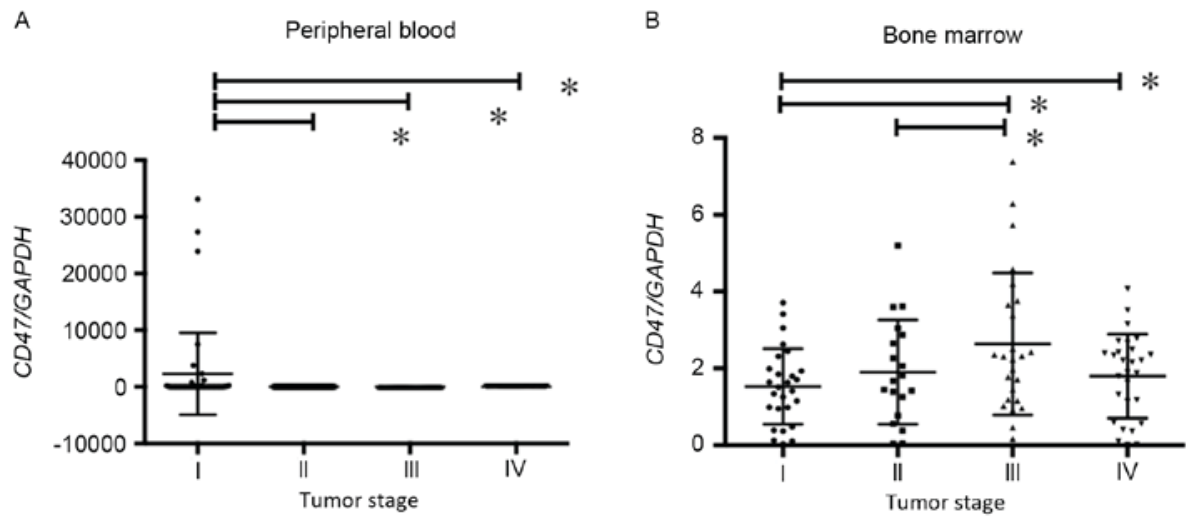

Figure 3. Association between CD47 mRNA expression in the (A) peripheral blood or (B) bone marrow and the tumor clinical stage. "P<0.05. CD47, integrin-associated protein.

\section{Discussion}

In the present study, the localization of CD47 protein was confirmed in normal tissue and primary gastric tumor tissue using immunohistochemical staining. Despite initial expectations, no statistically significant association was observed between primary tumor CD47 expression and clinicopathological factors, which has previously been detected in hematological malignancies $(7,17,18)$ and breast cancer (10). The Kaplan-Meier overall survival curve revealed that the survival rate of the high CD47 expression group was decreased compared with the low CD47 expression group from 3 years following surgery. This indicates that high CD47 expression is associated with late phase metastasis or recurrence of gastric cancer; however, the difference in survival between the two groups did not reach a statistically significant level. By contrast, low expression of CD47 in PB samples was significantly associated with increased tumor depth, lymphatic invasion, lymph node metastasis and clinical tumor stage. Conversely, high expression of CD47 in BM samples was associated with increased tumor depth, lymphatic invasion, lymph node metastasis and clinical stage. It was also revealed that CD47 mRNA expression was highest in the lymphocyte fraction in PB and BM samples. The contradiction of the CD47 
Table III. Association between CD47 mRNA expression in bone marrow and the clinicopathological characteristics of patients with gastric cancer.

CD47 expression group

\begin{tabular}{lccc} 
Clinicopathological characteristic & High & Low & P-value \\
\hline Total, $\mathrm{n}$ & 97 & 98 & - \\
Age, years, mean \pm SD & $61.2 \pm 1.21$ & $62.5 \pm 1.21$ & 0.445 \\
Age distribution, $\mathrm{n} \geq 70 /<70$ years $(\% \geq 70)$ & & & \\
Gender, male/female $(\%$ male) & $59 / 38(60.8)$ & $73 / 25(74.5)$ & $0.0408^{\mathrm{a}}$ \\
Tumor factors & & & \\
Tumor size, mm, mean \pm SD & $77.23 \pm 48.11$ & $65.87 \pm 47.63$ & 0.101 \\
Tumor size distribution, $\mathrm{n} \geq 50 /<50$ mm $(\% \geq 50)$ & $60 / 37(61.9)$ & $53 / 45(54.1)$ & 0.31 \\
Depth of tumor, $\mathrm{n}$ T1-2/T3-4 $(\%$ T1-2) & $28 / 69(28.7)$ & $46 / 52(47.4)$ & $0.0076^{\mathrm{a}}$ \\
Tumor type, $\mathrm{n}$ type $0-1 / 2-5(\% 0-1)$ & $60 / 37(61.9)$ & $49 / 49(50.0)$ & 0.220 \\
Lymphatic invasion, $\mathrm{n}+/-(\%+)$ & $63 / 34(65.2)$ & $44 / 54(45.2)$ & $0.0071^{\mathrm{a}}$ \\
Vascular invasion, $\mathrm{n}+/-(\%+)$ & $20 / 77(20.9)$ & $24 / 74(24.7)$ & 0.545 \\
Metastatic factors & & & \\
Lymph node metastasis, $\mathrm{n}+/-(\%+)$ & $74 / 23(76.4)$ & $53 / 45(54.3)$ & $0.0015^{\mathrm{a}}$ \\
Liver metastasis, $\mathrm{n}+/-(\%+)$ & $1 / 96(1.0)$ & $2 / 96(2.0)$ & 0.563 \\
Peritoneal (micro) metastasis, $\mathrm{n}+/-(\%+)$ & $17 / 80(17.5)$ & $16 / 82(16.5)$ & 0.848 \\
Peritoneal $($ macro) metastasis, $\mathrm{n}+/-(\%+)$ & $10 / 87(10.3)$ & $5 / 93(5.1)$ & 0.169 \\
Distant metastasis, $\mathrm{n}+/-(\%+)$ & $0 / 97(0.0)$ & $5 / 93(5.1)$ & $0.0081^{\mathrm{a}}$ \\
Clinical stage, $\mathrm{n} \mathrm{I} / \mathrm{II}-\mathrm{IV}(\% \mathrm{I})$ & $14 / 83(14.9)$ & $33 / 65(34.0)$ & $0.0019^{\mathrm{a}}$ \\
\hline
\end{tabular}

${ }^{\mathrm{a}} \mathrm{P}<0.05$. CD47, integrin-associated protein; $\mathrm{SD}$, standard deviation.
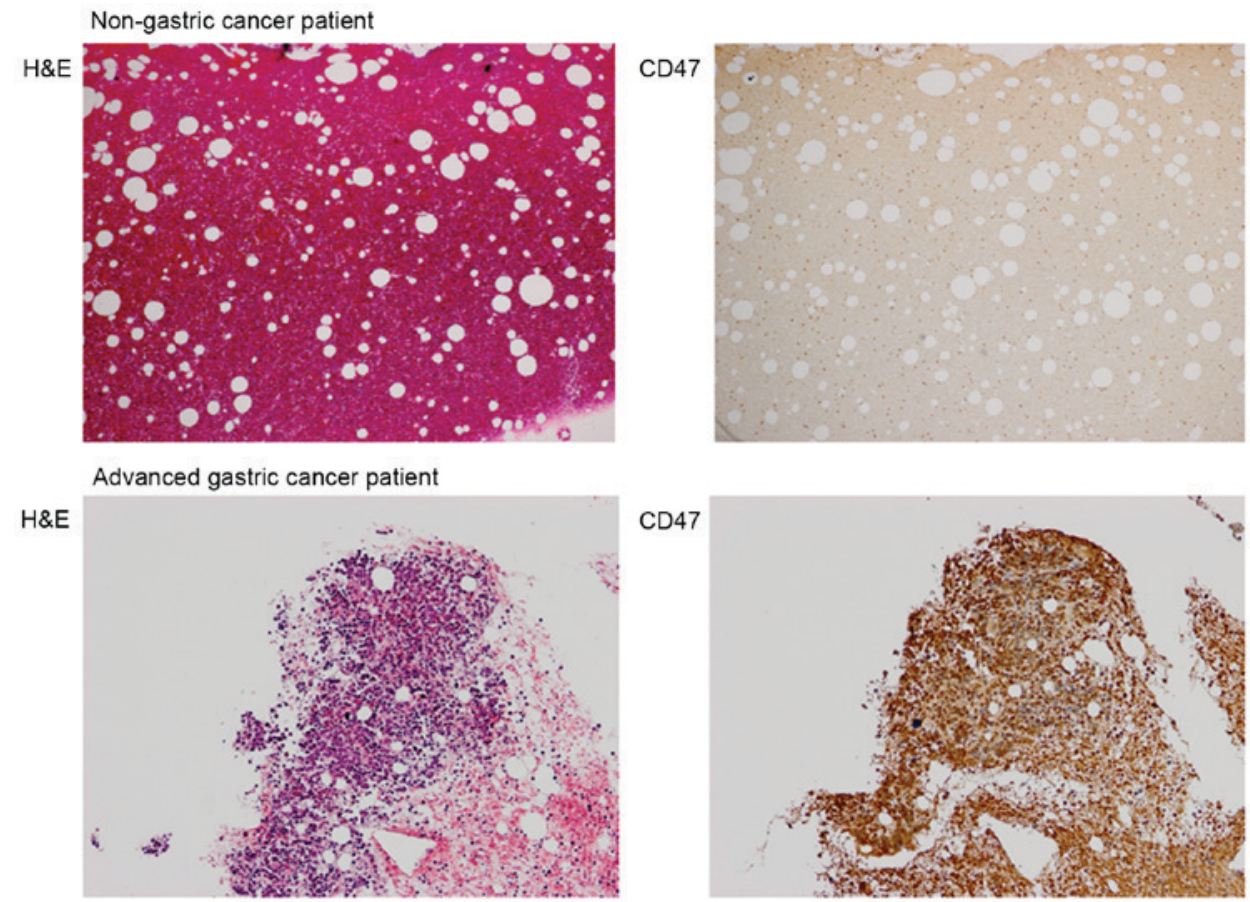

Figure 4. H\&E and immunohistochemical staining for CD47 in the bone marrow collected from a patient without cancer and a patient with stage IV gastric cancer. The left panels are H\&E stained; right panels are immunohistochemically stained for CD47. Upper panels, x20 magnification; lower panels, x40 magnification. H\&E, hematoxylin \& eosin; CD47, integrin-associated protein.

expression pattern between the primary tumor, $\mathrm{PB}$ and $\mathrm{BM}$ must be explained.
In normal gastric tissue, CD47 expression was limited to the lower layer, termed the fundic glands, in the current 


\section{Hypothesis}

Normal or early cancer stage

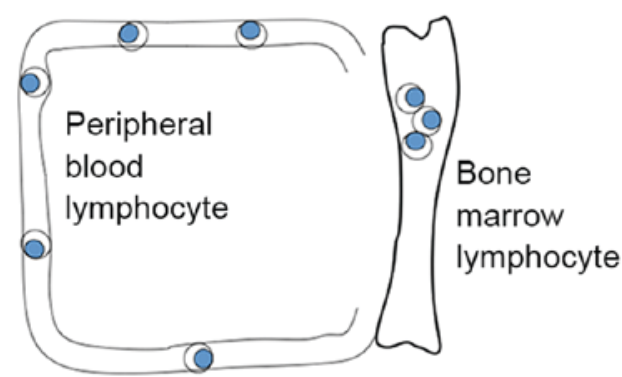

Cancer progression

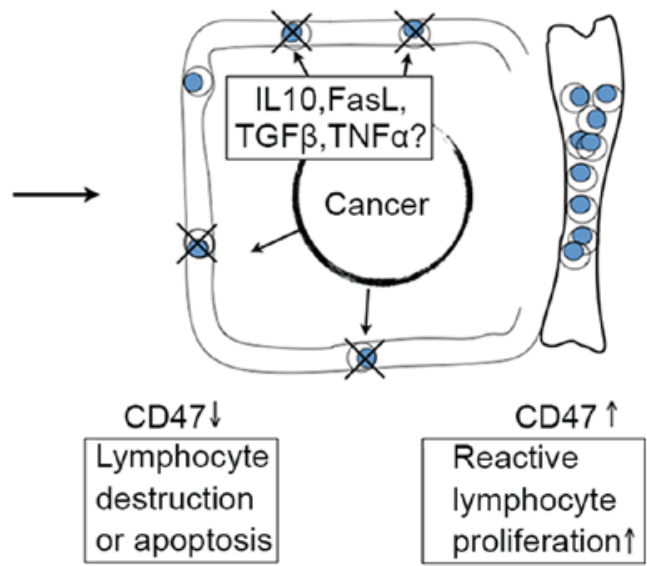

Figure 5. Hypothetical schema explaining the results of the present study. CD47, integrin-associated protein; IL10, interleukin-10; FasL, tumor necrosis factor ligand superfamily member 6 ; TGF $\beta$, transforming growth factor $\beta ; \mathrm{TNF} \alpha$, tumor necrosis factor $\alpha$.

study. The expression of CD47 is particularly observed from the portion of isthmus to the base of the glands. The fundic glands contain gastric mucosal stem cells and progenitor cells (19-21). By expressing CD47, the stem cells evade macrophage phagocytosis and elimination (6), and therefore the CD47 expression observed in normal mucosa is consistent with previous reports.

In primary gastric tumors, there was no indication of any statistical association between CD47 mRNA expression and clinicopathological factors. In the immunohistochemical staining analysis, a varied pattern of staining was observed, but there was no definitive pattern indicating that CD47 protein expression contributes to macrophage phagocytosis evasion. In addition, it was difficult to objectively evaluate which staining patterns were CD47-dominant or -negative; thus, it may not be appropriate to evaluate CD47 expression using immunohistochemical staining analysis in cancer. To make objective evaluations, the mRNA expression level of CD47 was calculated; however, bulky samples were used for mRNA collection in the present study, such that the heterogeneity of the tumor as a whole may have been erased.

In PB, upregulation of CD47 mRNA expression was associated with improved clinicopathological factors and early stage disease. By contrast, the association between CD47 expression and clinicopathological factors was the opposite in the BM. CD47 mRNA was expressed in the lymphocyte fraction in $\mathrm{PB}$ and $\mathrm{BM}$ samples, and this expression was low in the circulating tumor cell fraction, indicating that CD47 mRNA expression reflects lymphocytes but not cancer cells. Downregulation of CD47 is presumed to be caused by a reduction in lymphocytes. Several studies have indicated that the neutrophil:lymphocyte ratio (NLR) is a prognostic marker for gastric cancer (22-27). In early gastric cancer, cancer-associated mortality has been reported to be significantly more frequent among patients with an elevated NLR (>2) (28). In advanced gastric cancer, upregulation of the NLR to $>2.5$ is associated with a poor prognosis (29). The cause of the elevated NLR in this study was due to a surge in neutrophils and decline in lymphocytes, which is consistent with the decline in CD47 expression observed in the present study.

Lymphocyte reduction may be explained partially by the effect of an elevated release of several biological factors and cytokines into the PB from cancer tissues. Saito et al (30) demonstrated that a decline in natural killer (NK) cells, a type of lymphocyte, was caused by elevated tumor necrosis factor ligand superfamily member 6 expression, which shortened the lifespan of circulating NK cells by inducing their apoptosis. By contrast, the elevation of lymphocytes in BM is potentially the result of reactive proliferation in response to decreased lymphocytes in the PB (Fig. 5).

The reason for the discrepancy between the findings for CD47 expression in gastric cancer compared with blood and breast cancer requires further analysis. In the present study, the level of CD47 expression was investigated using PB samples, so that the expression level of CD47 would be representative of all cells in the blood, including lymphocyte and RBCs expressing CD47. Future studies should also collect the circulating tumor cells for analysis of CD47 expression levels.

Recently, Yoshida et al (31) reported that the survival rate of patients with CD47-expressing gastric cancer was significantly worse compared with that of patients with CD47-negative gastric cancer, using immunohistochemical staining analysis. In the present study, increased CD47 mRNA expression in the primary gastric tumor was not an adverse prognostic factor. This suggests that there may be post-transcriptional differences that cause CD47 protein expression to be higher, such as disruption of the protein degradation or transportation.

To the best of our knowledge, this is the first study to analyze CD47 expression in primary tumors, PB and BM simultaneously in patients with gastric cancer. The results indicate that CD47 expression in the PB and BM may serve as a marker to analyze the immunological function of patients with gastric cancer; however, to elucidate the significance of CD47 in gastric cancer is a complicated task. Therefore, if anti-CD47 antibody treatment is considered for gastric cancer, it may not be appropriate to make the decision based on the measurement of CD47 for this disease. 


\section{References}

1. Siegel R, Naishadham D and Jemal A: Cancer statistics, 2013. CA Cancer J Clin 63: 11-30, 2013.

2. Katanoda K, Hori M, Matsuda T, Shibata A, Nishino Y, Hattori M, Soda M, Ioka A, Sobue T and Nishimoto H: An updated report on the trends in cancer incidence and mortality in Japan, 1958-2013. Jpn J Clin Oncol 45: 390-401, 2015.

3. Matsuda T, Ajiki W, Marugame T, Ioka A, Tsukuma $\mathrm{H}$ and Sobue T: Research group of population-based cancer registries of Japan: Population-based survival of cancer patients diagnosed between 1993 and 1999 in Japan: A chronological and international comparative study. Jpn J Clin Oncol 41: 40-51, 2011.

4. Mawby WJ, Holmes CH, Anstee DJ, Spring FA and Tanner MJ: Isolation and characterization of CD47 glycoprotein: A multispanning membrane protein which is the same as integrin-associated protein (IAP) and the ovarian tumour marker OA3. Biochem J 304: 525-530, 1994.

5. Reinhold MI, Lindberg FP, Plas D, Reynolds S, Peters MG and Brown EJ: In vivo expression of alternatively spliced forms of integrin-associated protein (CD47). J cell Sci 108: 3419-3425, 1995.

6. Oldenborg PA, Zheleznyak A, Fang YF, Lagenaur CF, Gresham HD and Lindberg FP: Role of CD47 as a marker of self on red blood cells. Science 288: 2051-2054, 2000.

7. Jaiswal S, Jamieson CH, Pang WW, Park CY, Chao MP, Majeti R, Traver D, van Rooijen N and Weissman IL: CD47 is upregulated on circulating hematopoietic stem cells and leukemia cells to avoid phagocytosis. Cell 138: 271-285, 2009.

8. Chao MP, Weissman IL and Majeti R: The CD47-SIRP $\alpha$ pathway in cancer immune evasion and potential therapeutic implications. Curr Opin Immunol 24: 225-232, 2012.

9. Chan KS, Espinosa I, Chao M, Wong D, Ailles L, Diehn M, Gill H, Presti J Jr, Chang HY, van de Rijn M, et al: Identification, molecular characterization, clinical prognosis, and therapeutic targeting of human bladder tumor-initiating cells. Proc Natl Acad Sci USA 106: 14016-14021, 2009.

10. Nagahara M, Mimori K, Kataoka A, Ishii H, Tanaka F Nakagawa T, Sato T, Ono S, Sugihara K and Mori M: Correlated expression of CD47 and SIRPA in bone marrow and in peripheral blood predicts recurrence in breast cancer patients. Clin Cancer Res 16: 4625-4635, 2010.

11. Liu X, Pu Y, Cron K, Deng L, Kline J, Frazier WA, Xu H, Peng H, Fu YX and Xu MM: CD47 blockade triggers T cell-mediated destruction of immunogenic tumors. Nat Med 21: 1209-1215, 2015.

12. Soto-Pantoja DR, Stein EV, Rogers NM, Sharifi-Sanjani M, Isenberg JS and Roberts DD: Therapeutic opportunities for targeting the ubiquitous cell surface receptor CD47. Expert Opin Ther Targets 17: 89-103, 2013.

13. Japanese Gastric Cancer Association: Japanese gastric cancer treatment guidelines 2014 (ver. 4). Gastric Cancer 20: 1-19, 2017.

14. Chomczynski P: A reagent for the single-step simultaneous isolation of RNA, DNA and proteins from cell and tissue samples. Bio Techniques 15: 532-534, 536-537, 1993.

15. Inoue H, Mori M, Honda M, Li J, Shibuta K, Mimori K, Ueo H and Akiyoshi T: The expression of tumor-rejection antigen 'MAGE' genes in human gastric carcinoma. Gastroenterology 109: $1522-1525,1995$.
16. Livak KJ and Schmittgen TD: Analysis of relative gene expression data using real-time quantitative PCR and the 2(-Delta Delta C(T)) Method. Methods 25: 402-408, 2001

17. Majeti R, Chao MP, Alizadeh AA, Pang WW, Jaiswal S, Gibbs KD Jr, van Rooijen N and Weissman IL: CD47 is an adverse prognostic factor and therapeutic antibody target on human acute myeloid leukemia stem cells. Cell 138: 286-299, 2009.

18. Chao MP, Alizadeh AA, Tang C, Jan M, Weissman-Tsukamoto R, Zhao F, Park CY, Weissman IL and Majeti R: Therapeutic antibody targeting of CD47 eliminates human acute lymphoblastic leukemia. Cancer Res 71: 1374-1384, 2011.

19. Hoffmann W: Regeneration of the gastric mucosa and its glands from stem cells. Curr Med Chem 15: 3133-3144, 2008.

20. Hoffmann W: Stem cells, self-renewal and cancer of the gastric epithelium. Curr Med Chem 19: 5975-5983, 2012.

21. Lee ER and Leblond CP: Dynamic histology of the antral epithelium in the mouse stomach: II. Ultrastructure and renewal of isthmal cells. Am J Anat 172: 205-224, 1985.

22. Shimada H, Takiguchi N, Kainuma O, Soda H, Ikeda A, Cho A, Miyazaki A, Gunji H, Yamamoto $\mathrm{H}$ and Nagata M: High preoperative neutrophil-lymphocyte ratio predicts poor survival in patients with gastric cancer. Gastric Cancer 13: 170-176, 2010.

23. Balta S, Unlu M, Arslan Z and Demirkol S: Neutrophil-tolymphocyte ratio in prognosis of gastric cancer. J Gastric Cancer 13: 196-197, 2013

24. Cho IR, Park JC, Park CH, Jo JH, Lee HJ, Kim S, Shim CN, Lee H, Shin SK, Lee SK and Lee YC: Pre-treatment neutrophil to lymphocyte ratio as a prognostic marker to predict chemotherapeutic response and survival outcomes in metastatic advanced gastric cancer. Gastric Cancer 17: 703-710, 2014

25. Petrillo A, Laterza MM, Ventriglia J, Savastano B, Tirino G, Pompella L, Martinelli E, Morgillo F, Orditura M, Ciardiello F and De Vita F: Prognostic implications of baseline neutrophil-lymphocyte ratio (NLR) and platelet-lymphocyte ratio (PLR) in metastatic gastric cancer (GC) patients. Ann Oncol 27: (Suppl 6): 652P, 2016

26. Zhang X, Zhang W and Feng LJ: Prognostic significance of neutrophil lymphocyte ratio in patients with gastric cancer: A meta-analysis. PLoS One 9: e111906, 2014.

27. Lee S, Oh SY, Kim SH, Lee JH, Kim MC, Kim KH and Kim HJ: Prognostic significance of neutrophil lymphocyte ratio and platelet lymphocyte ratio in advanced gastric cancer patients treated with FOLFOX chemotherapy. BMC Cancer 13: 350, 2013.

28. Hirashima M, Higuchi S, Sakamoto K, Nishiyama T and Okada H: The ratio of neutrophils to lymphocytes and the phenotypes of neutrophils in patients with early gastric cancer. J Cancer Res Clin Oncol 124: 329-334, 1998.

29. Yamanaka T, Matsumoto S, Teramukai S, Ishiwata R, Nagai Y and Fukushima M: The baseline ratio of neutrophils to lymphocytes is associated with patient prognosis in advanced gastric cancer. Oncology 73: 215-220, 2007.

30. Saito H, Takaya S, Osaki T and Ikeguchi M: Increased apoptosis and elevated Fas expression in circulating natural killer cells in gastric cancer patients. Gastric Cancer 16: 473-479, 2013.

31. Yoshida K, Tsujimoto H, Matsumura K, Kinoshita M, Takahata R, Matsumoto Y, Hiraki S, Ono S, Seki S, Yamamoto J and Hase K: CD47 is an adverse prognostic factor and a therapeutic target in gastric cancer. Cancer Med 4: 1322-1333, 2015. 